\title{
Design of Multiple Vehicle Video Monitor System Based on MST703
}

\author{
Zhongmin $\mathrm{LI}^{1,}$, , Zhiwei Fan ${ }^{2, \mathrm{~b}}$ \\ ${ }^{1}$ School of Information Engineering, Nanchang Hangkong University, Nanchang, 330063, China \\ ${ }^{2}$ School of Information Engineering, Nanchang Hangkong University, Nanchang, 330063, China \\ aemail: zhongmli@163.com, bemail: 2926135994@qq.com
}

\section{Keywords: Video Monitor; MST703; Camera; Liquid Crystal Display}

\begin{abstract}
With the development of electronic technology and road traffic, more and more electronic equipments are used in automobiles and vehicle monitoring system. Car rear monitoring system can eliminate the visual blind spot, provide the driver wider perspective and make driving safer. Now car video monitoring system has become a focus of research. In this paper, we choose MST703 as the core processing chip, achieve the acquisition of multi-channel video signal processing, display and switch. In this system, analog cameras are used for video signal collection, and MST703 chip is used to process video signal, control all of the modules of the system, and display on the LCD screen. And multiplex video signal switching works in the form of software implementation in this system.
\end{abstract}

\section{Introduction}

With the development of highway transportation and the popularization of cars, the car has played an increasingly important role in daily life. However, with the increasing of the car, there are more and more traffic accidents, so traffic safety is more and more getting the attention of people. In recent years, with the rapid development of electronic technology, computer technology and communication technology, information technologies constantly penetrate to the automobile industry. The vehicle's safety protection becomes a very popular research field. The future cars will rapidly develop in secure, intelligent and simple operation. The car is going to be a smart ride space [1]. Under this trend, multiple vehicle monitor system is more and more getting attention. It allows the driver to easily understand the car's front, rear and the situation of the car and makes driving more safe and comfortable. Especially rear view monitoring can greatly eliminate the visual blind area and make driving safer than before.

At present, there are many types of vehicle video monitoring systems which are implemented. There are mainly two types: one is respectively based on DSP+FPGA [2], and the other is based on ARM + Linux [3]. DSP is specially designed for the realization of digital processing of high-performance single chip processor and has the very high processing speed. DSP can be integrated video unit and interface within it, use the software realization of video decoding function, and support a variety of coding compression algorithm. ARM is a high-performance processor which is used widely. It can be integrated memory, on-chip resources and peripheral interfaces. It has fast speed of processing and supports all kinds of embedded software. Because Linux is open source and its function gradually improves, with the ARM to build hardware platform and the Linux operating system as the software platform of vehicle video monitoring system is also a common way.

In this paper, a new optimal design of multiple vehicle video monitor system based on MST703 is presented, in which specific integrated circuits MST703 is used as the key control chip. It is a dedicated video decoder chip and integrates some peripheral interfaces. It realizes the hardware method of video coding and its coding compression algorithm in the factory had been cured. Therefore, it has the advantage in cost and performance. The overall design is convenient and simple. 


\section{The Overall Design}

This design adopts MST703 as the core to control the collection of AD and data processing. We select analog cameras, which are responsible for collecting video image information. MST703 chip mostly supports four-way analog video signal input. Three pins of the analog camera receive power supply cathode, ground and MST703 chip analog signal input. MST703 chip is going to be analog-digital conversion, decoding [4], processing for input analog video signal. Finally, it transfers video to the touch screen and displays. Four-way video switch is implemented by means of software. The system total design is shown in Fig. 1.

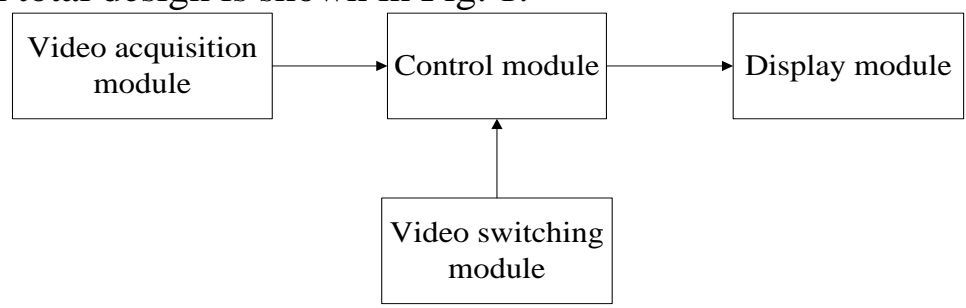

Fig.1.The system total design

\section{The Hardware Design}

This system is mainly composed of camera part, liquid crystal display part and MST703 control part.

System core control unit MST703. MST703 is a special video decoding chip which is produced by Aipuwei Electronic science. The MST703 is a high quality ASIC for NTSC/PAL/SECAM car TV application. It receives analog NTSC/PAL/SECAM CVBS and S-Video inputs from TV tuners, DVD or VCR sources, including weak and distorted signal, as well as analog YCbCr input from HDTV/SDTV system [5]. Automatic gain control (AGC) and 10-bit 3-channel A/D converters provide high resolution video quantization. With automatic video source and mode detection, users can easily switch and adjust variety of signal sources. Multiple internal adaptive PLLs precisely extract pixel clock from video source and perform sharp color demodulation. Built-in line-buffer supports adaptive 2-D comb-filter, 2-D sharpening, and synchronization more stable in a condense manner. The output format of MST703 supports 6-bit TTL/TCON and LVDS digital TFT-LCD modules.

Camera module. This design uses analog camera which type is SK-208B produced by the SANKE Company. The camera uses CMOS sensor. Its production is easy and the cost is low. It can achieve a higher level of integration and has the advantages of ultra-low power consumption. The output signal system of cameras is the PAL. It can be directly received the video input terminal of MST703 chip and realizes the data transmission. Working temperature is between $-10 \sim+50^{\circ} \mathrm{C}$. Working voltage is DC6V/200mA. System design is simple and convenient to use it, and can completely meet the design requirements.

Liquid crystal display module. At present, the domestic starts to use the LCD monitor which has many kinds, such as the STN and TFT. They each have advantages and disadvantages. This design adopts AT070TN92 TFT LCD of the INNOLUX Company. This display support $800 \times 480$ resolution and supporting the palette of display mode is 256 color (8 BPP). TFT LCD can greatly shorten the response time of screen and its response time is less than $80 \mathrm{~ms}$. Relative to the STN LCD, it can improve fuzzy screen flicker when the STN is continuous display, and effectively improve the dynamic images playing ability to make presenting the picture color saturation, actual effect and contrast very good. It is completely beyond the STN and is just slightly higher power consumption. It is currently the most mainstream type liquid crystal display (LCD), and is also very widely used in all kinds of products [7]. The system hardware principle diagram is shown in Fig. 2. 


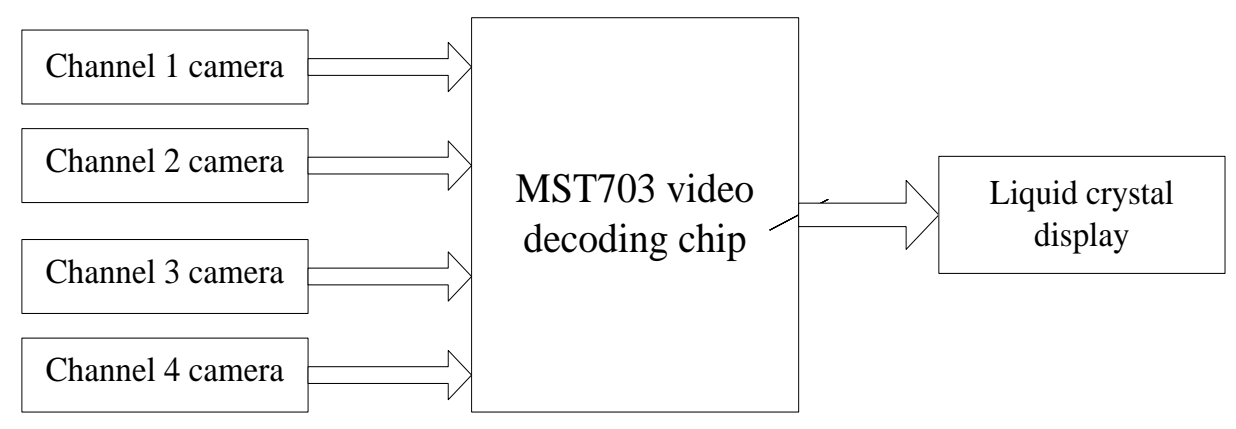

Fig.2. The system hardware principle diagram

\section{The Software Design}

The software design [8] is the focus of the realization of switching between multiple video and is also difficulty. In programming, when the user opens the system, the system will be initialized, open reversing interruption, and check whether the car is in reverse. If the car is in reverse, the system will forcibly switch the camera captured video signal in the back of the car and display on the LCD. The user can easily understand the situation in the rear of the car to prevent danger. If the car is not in reverse, the user can arbitrarily switch video signal source through the buttons on the LCD screen interface. Switching among the video source is achieved by software. Software design flow chart is shown in Fig. 3.

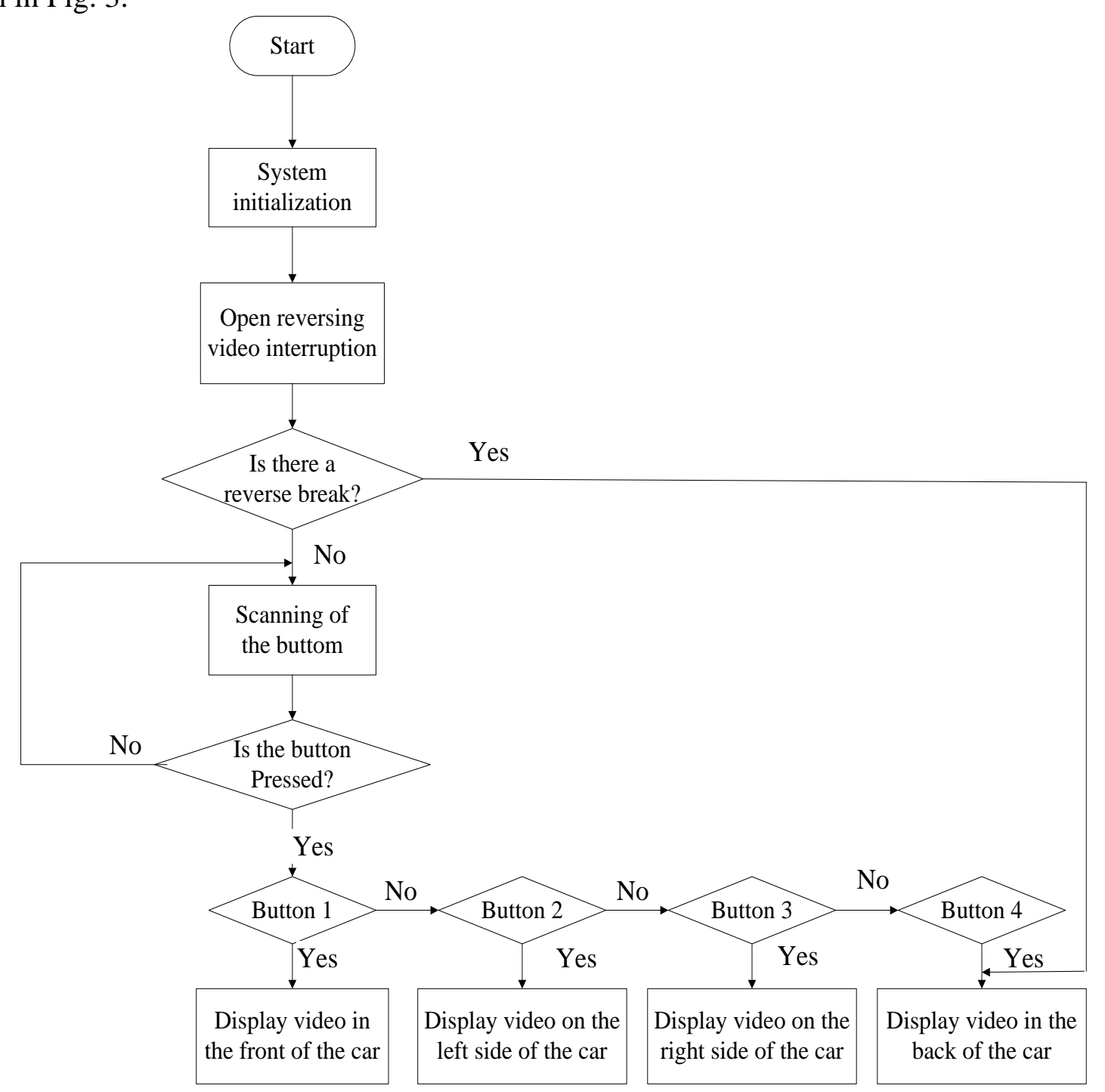

Fig.3 The system software flow chart 


\section{Conclusion}

In this paper, we use MST703 chip as the core to design a multi-channel video monitoring system. Users can directly operate on the LCD screen to realize mutual switch among the various videos. This system is not easy to be damaged and has high safety and long service life. The design has the characteristics of low cost, strong practicability and high application value.

\section{Acknowledgement}

In this paper, the research was sponsored by the Programs of the National Natural Science Foundation of China (Project No. 61263040 and No. 61162002), and the Scientific Research Foundation of Jiangxi Provincial Education Department in China (Project No. GJJ13528).

\section{References}

[1] Chending Feng. Talk vehicle monitoring market prospects Analysis: vehicle monitoring to create urban "new means of transport" [J]. Investment and Cooperation, 2014 (7):7.

[2] Guangsheng Li. Hardware system design of vehicle video processing based on DM642 [D]. Shijiazhuang Railway Institute, 2013.

[3] Junjun Fu, Qisong Chen. Design and implementation of remote video surveillance system based on ARM9+Linux [J]. Based Automation and Instrumentation, 2014 (4): 131-134.

[4] Guanqun Liu. Screen display optimization after video decoding [J]. Modern Modern Electronics Technique, 2013:36(5):161-162.

[5] Shazi Xi. Security in the world - Detailing and practice of intelligent network video monitoring technology [M]. Beijing: Tsinghua University Press, 2010: 3-8.

[6] Dongshan Wei. Complete manual for embedded Linux application development [M]. Beijing: People's Posts and Telecommunications Press, 2009: 242-243.

[7] Yufeng Gong. Software developments of LCD display [D]. Beijing University of Posts and Telecommunications, 2012.

[8] Chunyuan Bian, Zhiqiang Wang. MCS-51 microcontroller development of practical application subroutine [M]. Beijing: People's Posts and Telecommunications Press, 2005. 